\title{
Penetration in bimodal, polydisperse granular material
}

\author{
N. Kouraytem, ${ }^{1}$ S. T. Thoroddsen, ${ }^{1}$ and J. O. Marston ${ }^{2, *}$ \\ ${ }^{1}$ Division of Physical Sciences and Engineering, King Abdullah University of Science and Technology, \\ Thuwal 23955-6900, Kingdom of Saudi Arabia \\ ${ }^{2}$ Department of Chemical Engineering, Texas Tech University, Lubbock, Texas 79409, USA \\ (Received 31 March 2016; revised manuscript received 14 September 2016; published 7 November 2016)
}

\begin{abstract}
We investigate the impact penetration of spheres into granular media which are compositions of two discrete size ranges, thus creating a polydisperse bimodal material. We examine the penetration depth as a function of the composition (volume fractions of the respective sizes) and impact speed. Penetration depths were found to vary between $\delta=0.5 D_{0}$ and $\delta=7 D_{0}$, which, for mono-modal media only, could be correlated in terms of the total drop height, $H=h+\delta$, as in previous studies, by incorporating correction factors for the packing fraction. Bimodal data can only be collapsed by deriving a critical packing fraction for each mass fraction. The data for the mixed grains exhibit a surprising lubricating effect, which was most significant when the finest grains $\left[d_{s} \sim O(30) \mu \mathrm{m}\right]$ were added to the larger particles $\left[d_{l} \sim O(200-500) \mu \mathrm{m}\right]$, with a size ratio, $\epsilon=d_{l} / d_{s}$, larger than 3 and mass fractions over $25 \%$, despite the increased packing fraction. We postulate that the small grains get between the large grains and reduce their intergrain friction, only when their mass fraction is sufficiently large to prevent them from simply rattling in the voids between the large particles. This is supported by our experimental observations of the largest lubrication effect produced by adding small glass beads to a bed of large sand particles with rough surfaces.
\end{abstract}

DOI: 10.1103/PhysRevE.94.052902

\section{INTRODUCTION}

In many experiments, and indeed simulations, of granular impact phenomena, the key characteristic of the granular media is that it is monomodal, with a distinct peak size and a relatively narrow size distribution, whereas most naturally occurring granular media are polydisperse with large distributions in both size and shape [1,2]. In order to derive the key physical mechanisms and properties governing impact-related phenomena such as crater formation, a number of studies [3-6] have focused on a simple experiment-namely - the impact of a sphere onto a static granular bed in order to assess the penetration depth of the sphere and size of the resulting crater using model granular materials. In addition, a number of studies focus on the temporal evolution of forces acting within the granular medium and on the impactor [7-11], while others have paid attention to the ejecta formations in various impact experiments [12-16]. For shallow penetrations, where $\delta=O\left(D_{0}\right)$ [see Fig. 1(a)], the works of Durian and co-workers $[3,6,17,18]$ provide authoritative sources of the various scalings for this key measurement and can generally be summarized in terms of the impactor density, diameter, and fall height $\left(\rho_{0}, D_{0}\right.$, and $h$, respectively) as

$$
\delta-\delta_{0} \propto \rho_{0}^{1 / 2} D_{0}^{2 / 3} h^{1 / 2},
$$

showing that the depth varies linearly with the impact speed $V_{0} \sim h^{1 / 2}$. Here, $\delta_{0} \equiv \delta\left(V_{0}=0\right)$. Similarly, in terms of the total drop distance, $H=h+\delta$,

$$
\delta \propto\left(\rho_{0} / \rho_{g} \mu^{2}\right)^{1 / 2} D_{0}^{2 / 3} H^{1 / 3},
$$

where $\rho_{g}$ indicates the bulk density of the granular bed and $\mu=\tan \theta_{r}$ is the friction coefficient, where $\theta_{r}$ is the angle of

*Corresponding author: jeremy.marston@ttu.edu repose of the granular material. de Bruyn and Walsh [19] used various sizes of glass beads and initial packing fractions to elucidate the influence of the bulk density of the bed, finding that the correction factor $\left(1-\phi / \phi_{c}\right)$ was needed to collapse their data for $\delta$ versus $H$ in Eq. (2). Here $\phi$ is the packing fraction and $\phi_{c}$ is a critical packing fraction [8] (close to the random close packing limit). Scalings for deeper penetrations generally take the form $\delta / D_{0} \propto\left(\rho_{0} / \rho_{g}\right)^{\beta}\left(H / D_{0}\right)^{\alpha}$, where both exponents are found to be dependent on the geometry of the container and, in particular, the container-to-sphere diameter ratio [20]. Recently, the effect of cohesion by mixing glass beads with water was studied [21,22], showing that the penetration depth exhibited a nonmonotonic dependence on the initial moisture content in the granular bed, whereby the cohesion from interparticle liquid bridges can play a dual role of creating a lower bulk density but also more internal friction, depending on the grain size and impact speed. In this sense, altering the composition of the bed can either "lubricate" or "stiffen" the granular bed. In this communication, we thus seek to extend the premise of lubricating the granular media [21,22] and pose the fundamental question: can smaller grains be used to lubricate the impact and increase penetration into granular material?

\section{EXPERIMENTAL SETUP}

To render a bimodal granular material, we mix two discrete size ranges together from a selection of four sizes: three glass bead media used have volume-based mean diameters of $d_{4,3}=520,178$, and $31 \mu \mathrm{m}$, respectively, with $\left(d_{10}, d_{50}, d_{90}\right)=$ $(441,493,564),(127,174,234)$ and $(19,30,46) \mu \mathrm{m}$, where $d_{10}$, $d_{50}$, and $d_{90}$ correspond to the 10th, 50th, and 90th percentiles for size fractions, respectively. All three have the same approximate material density $\rho_{g} \approx 2500 \mathrm{~kg} / \mathrm{m}^{3}$ and mean circularity $C=4 \pi A / P^{2} \approx 0.96$, where $A$ and $P$ are the area and perimeter of the grain, respectively. A single quantitative 


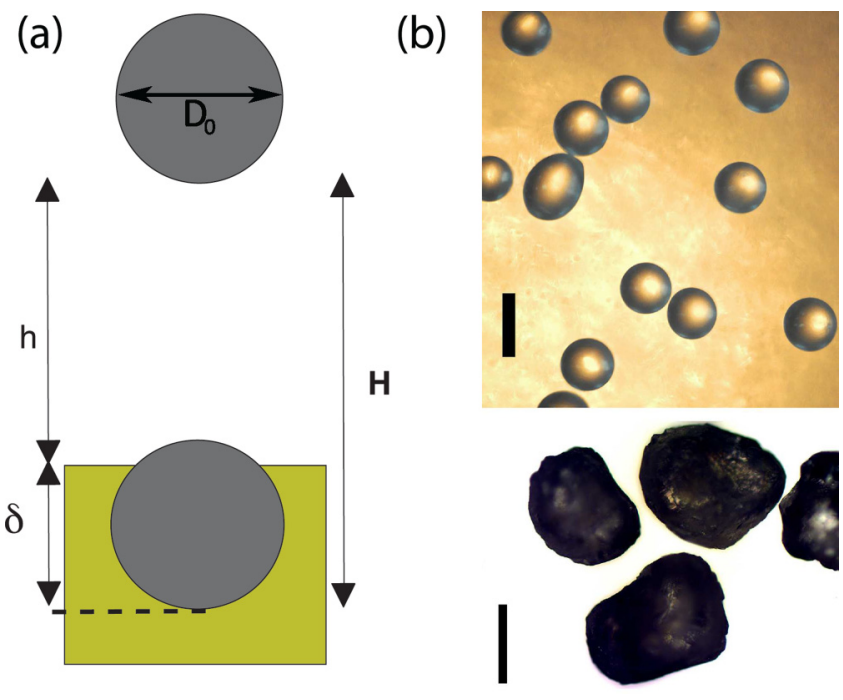

FIG. 1. (a) Schematic showing graphical definitions of the release height, $h$; the penetration depth, $\delta$; and the total drop height, $H=h+\delta$, for this experiment. (b) Comparison of grain roughness between glass beads (top, scale bar, $500 \mu \mathrm{m}$ ) and Ottawa sand (bottom, scale bar, $250 \mu \mathrm{m}$ ).

measure of polydispersity can be given by $\Delta S=\left(d_{90}-\right.$ $\left.d_{10}\right) / d_{50}=0.25,0.61$ and 0.9 , respectively, showing that the smallest beads have a higher relative polydispersity [13].

In addition, Ottawa sand (Fisher Scientific) was used with a mean size of $d_{m}=350 \mu \mathrm{m}, \Delta S=0.33$, density $\rho_{g} \approx 2.65 \mathrm{~kg} / \mathrm{m}^{3}$, and mean circularity $C \approx 0.81$, indicating a more irregular shape [see Fig. 1(b)].

The angle of repose was measured by slowly flowing a stream of the granular material in question through a funnel and measuring the value of the angle formed with the horizontal immediately before the cone-shaped pile would break and avalanche. The angle of repose was found to be $\theta_{r}=24^{\circ}$, $30^{\circ}$, and $35^{\circ}$ for the 520-, 178-, and $31-\mu \mathrm{m}$ glass beads, respectively, while the mixtures all exhibited values of $\theta_{r}$ between $33^{\circ}$ and $38^{\circ}$.

For reference trials with the single size ranges the grains are poured into a square container $\left(D_{c}=9 \mathrm{~cm}, H_{c}=20 \mathrm{~cm}\right)$ and then either scraped level or tapped for $1 \mathrm{~min}$ and then scraped level. We then release a steel $\left(\rho_{0}=7800 \mathrm{~kg} / \mathrm{m}^{3}\right)$ or tungsten $\left(\rho_{0}=14000 \mathrm{~kg} / \mathrm{m}^{3}\right)$ sphere with a diameter of $D_{0}=2 \mathrm{~cm}$ from set release heights of $h \leqslant 1.6 \mathrm{~m}$ to impact on the granular beds with impact speed $V_{0} \approx \sqrt{2 g h}$, which is measured using a high-speed video camera. The penetration depth $\delta$ is measured from the initial bed surface to the bottom of the sphere and the total drop height is defined as $H=h+\delta$. These are shown graphically in Fig. 1(a). Note that $D_{C} / D_{0}=4.5$, which is below the critical ratio of 5 identified by Seguin et al. [20]. However, this is fixed for all experiments; thus the context of our analysis and discussion is independent of geometrical influence.

The larger grains are mixed with the smaller grains in numerous set mass fractions of $M=m_{\text {small }} /\left(m_{\text {large }}+m_{\text {small }}\right)$ between 2.5 and $70 \%$. For the bimodal mixtures, we do not use tapping in order to minimize any effects of size segregation. To increase the packing fractions of these mixtures, we overfill the container and compress the surface with a weighted plate as in previous studies [13].

\section{RESULTS}

\section{A. Monodisperse material}

Figure 2 shows the raw data for the penetration depth versus the impact speed for all three single size ranges of glass beads used in the experiment: Figure 2(a) for the lower packing fractions obtained by pouring the beads loosely into the container and Fig. 2(b) for the higher packing fractions obtained by tapping. These trends, while linear and monotonic, reveal some interesting basic observations. First, in Fig. 2(a), there is little difference between the two larger grain sizes (520 and $178 \mu \mathrm{m}$ ) with $\phi \approx 0.6$, while we observe at least a twofold increase in penetration depth for the finest grains with a lower packing fraction of $\phi \approx 0.5$. In contrast, for the higher packing fractions in Figure 2(b), all three grain sizes exhibit very similar penetration depths, indicating that particle size becomes less important at higher initial packing fractions. We may interpret these observations in the context of a critical packing fraction, $\phi^{*} \approx 0.6$, found by Umbanhower and Goldman [8] and Royer et al. [23], whereby the impact can bring about local fluidization for $\phi>\phi^{*}$, but local compaction for $\phi<\phi^{*}$, which may partly explain the slightly deeper penetration for $d_{4,3}=520 \mu \mathrm{m}$ at $\phi=0.64$ than for $d_{4,3}=$ $31 \mu \mathrm{m}$ at $\phi=0.58$ for the compacted grain in Fig. 2(b), the effect of $\Delta S$ notwithstanding. Air-mediated effects (e.g., Refs. [24,25]) may also be important for the finest grains at low packing fraction, since it is known that the interstitial air plays a significant role on the drag on a sphere. This was best demonstrated by Royer et al. using x-ray imaging [26], showing drag increases as the ambient pressure decreases.

In all cases, we find that the data exhibits a linear dependence on impact speed, which can be modeled simply by $\delta=\delta_{0}+\alpha_{1} V_{0}$, where $\delta_{0}$ represents the penetration depth for zero release height (i.e., where the linear fit cuts the $y$ axis) and $\alpha_{1}$ is a constant for each data set with units of time. Physically, this could be thought of as an average "stopping time." These trend lines have been included in the plots. For all eight data sets, we find that $\delta_{0} \approx 0.8-1.5 \mathrm{~cm}$, while the slope $\alpha_{1}$ varies considerably from $0.59 \mathrm{~s}$ for the higher packing fraction to $3.97 \mathrm{~s}$ for the lower packing fraction, both with the finest grains $\left(d_{4,3}=31 \mu \mathrm{m}\right)$. In a fashion similar to that of de Bruyn and Walsh [19], upon inspection of the slopes $\alpha_{1}$ as a function of $\phi$, we can deduce the critical value $\phi_{c}=64.5 \%$, very close to the random close packing fraction $\phi_{\text {rcp }} \approx 64 \%$. Using the correction factor $\left(1-\phi / \phi_{c}\right)$, we show that the data can be approximately described by the scaling $\delta \sim H^{1 / 3}$ observed previously $[6,17,19]$, as shown in Fig. 2(c), the effects of air notwithstanding.

\section{B. Bidisperse material}

Figure 3 plots the equivalent penetration data obtained for three different bidisperse mixtures over a wide range of mass fractions $M$ from $2.5 \%$ to $70 \%$. The two components in each mixture are of different size ratios: in Fig. 3(a) we mix 178- $\mu \mathrm{m}$ glass beads in $520-\mu \mathrm{m}$ glass beads, for a size ratio of 2.9 , while in Fig. 3(b) we use $31-\mu \mathrm{m}$ grains in $178-\mu \mathrm{m}$ grains giving a 

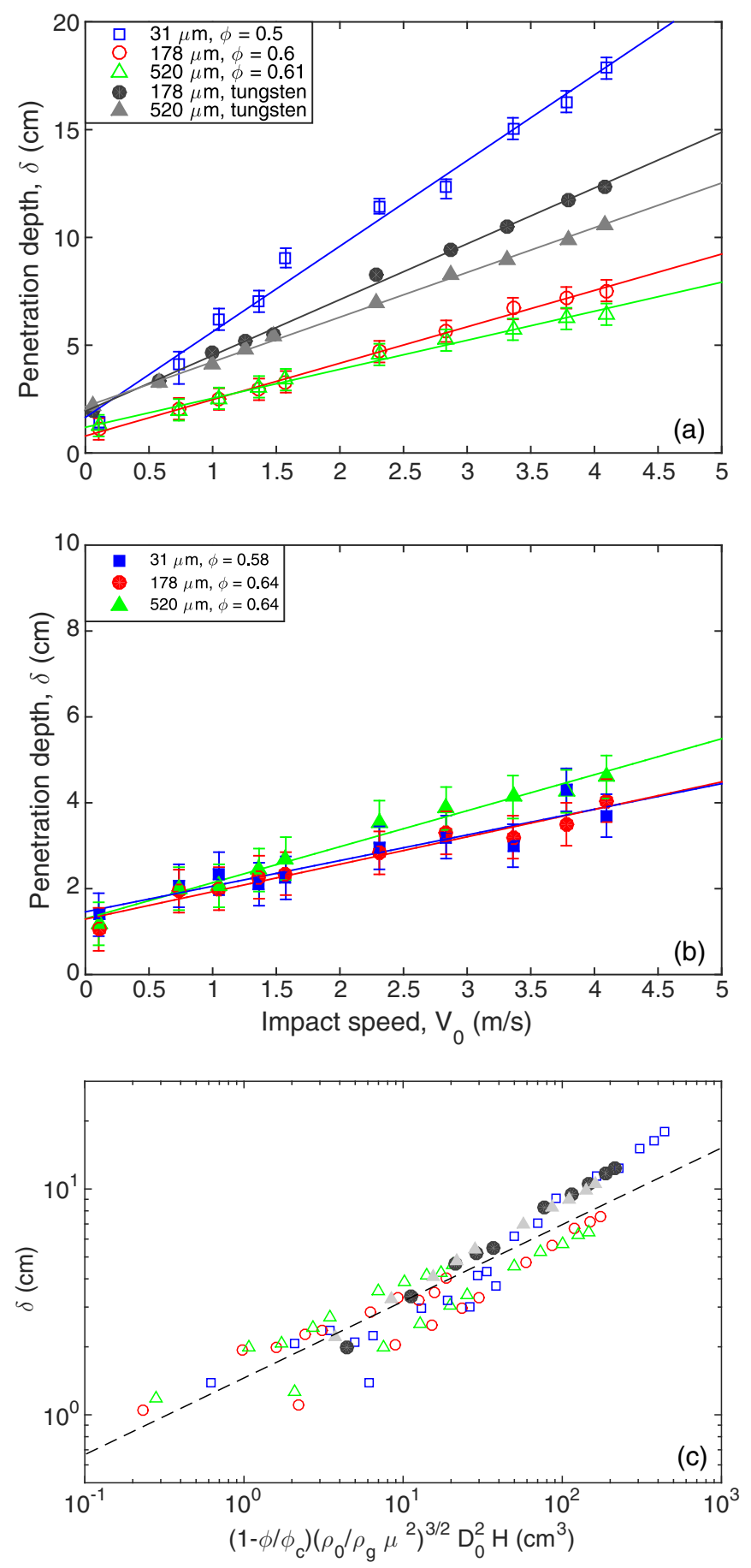

FIG. 2. Penetration depth versus impact speed for dry monodisperse glass beads either poured (a) or tapped (b). The legends indicate the grain size and the packing fraction. In panel (a), data include both steel and tungsten spheres. All the data from panels (a) and (b) are replotted versus the scaled drop height in panel (c), using the correction factor $1-\phi / \phi_{c}$ [19]. The dashed line indicates a slope of $1 / 3$.

size ratio of 5.7. Finally, in Figure 3(c) we study the largest size ratio of 17 , with a mixture of the smallest $31-\mu \mathrm{m}$ grains in the largest $520-\mu \mathrm{m}$ grains. In all three cases, the data are plotted alongside the trend lines for penetration into beds of the individual grains in the composition [from Fig. 2(a)].
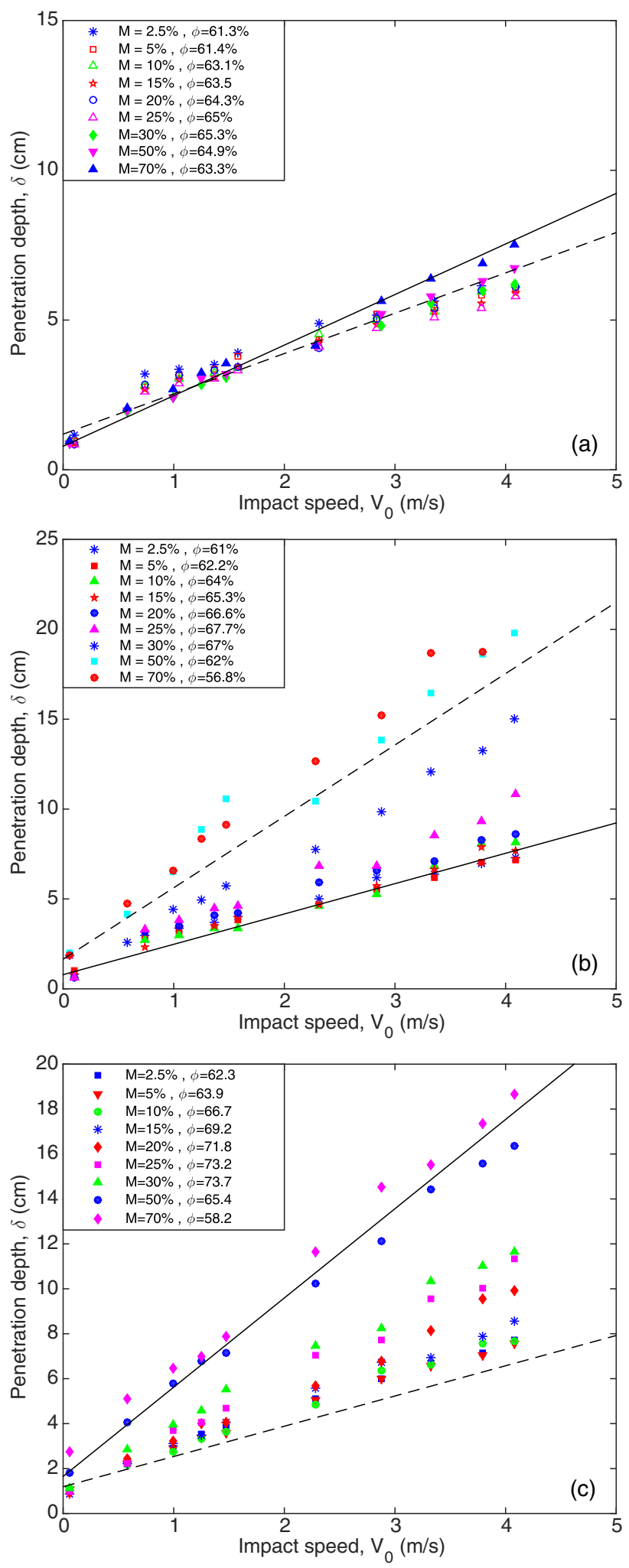

FIG. 3. Penetration depth versus impact speed for bidisperse mixtures: (a) $178-\mu \mathrm{m}$ beads mixed in $520-\mu \mathrm{m}$ beads, (b) $31-\mu \mathrm{m}$ beads mixed in $178-\mu \mathrm{m}$ beads, and (c) $31-\mu \mathrm{m}$ beads mixed with $520-\mu \mathrm{m}$ beads. The lines represent trend lines to the data of poured monodisperse grains shown in Fig. 2: (a) solid and dashed lines correspond to 178 and $520 \mu \mathrm{m}$, respectively; (b) solid and dashed lines correspond to 178 and $31 \mu \mathrm{m}$, respectively; and (c) solid and dashed lines correspond to 31 and $520 \mu \mathrm{m}$, respectively. 


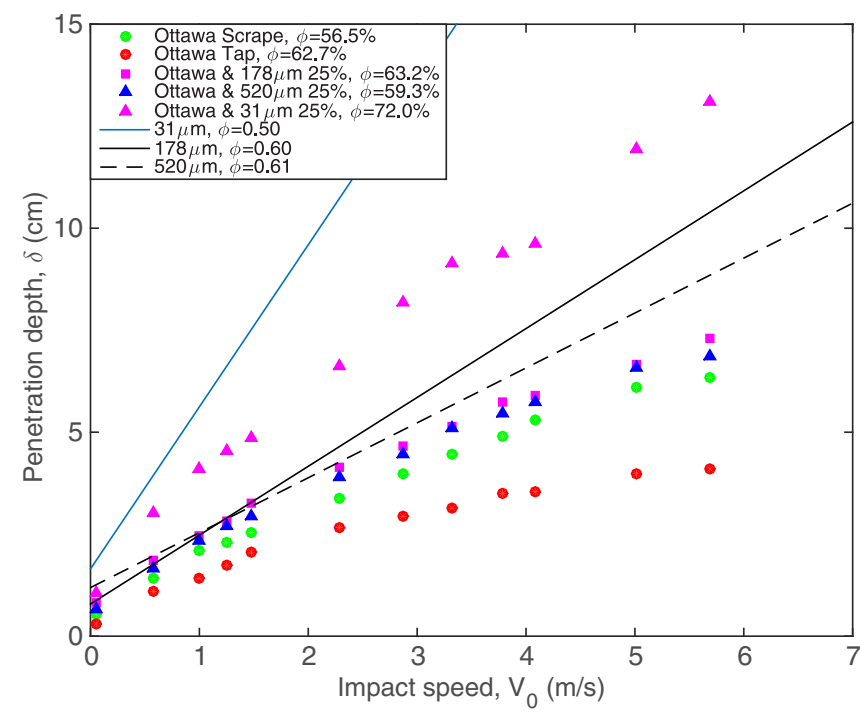

FIG. 4. Penetration depth versus impact speed for $350-\mu \mathrm{m}$ Ottawa sand mixed with the three glass beads $(M=25 \%)$.

For the smallest size ratio, in Fig. 3(a), the addition of the small grains does not significantly affect the penetration depth into the mixture, regardless of the mass fractions. This changes drastically when the size difference between the two grains becomes larger [see Figs. 3(b) and 3(c)]. For small mass fractions $(M \leqslant 20 \%)$ in Fig. 3(b) and $M \leqslant 15 \%$ in Fig. 3(c) there are no significant effects on the penetration depth. However, at larger mass fractions the smaller grains produce a strong effect as is clear by how quickly the penetration depth approaches that for the pure $31-\mu \mathrm{m}$ grains. Most striking is the data for $M=50 \%$ in Fig. 3(b), which shows the penetration depths actually exceeding the trend line for the smaller grains, despite having significantly higher packing fractions. The same trend is observable in Fig. 3(c), again 50\% reaches the pure smaller grain dynamics and $70 \%$ slightly overshoots the penetration depth for pure small grains.

\section{Irregular grains}

Based on the above results, we postulate that the principal mechanism for this lubrication is the smaller grains getting into the gaps between the larger grains, thereby propping them apart, promoting airflow fluidization and causing lubrication effects. If reduced friction between the larger grains is at play, then this effect should be even more dramatic for large grains which have rough surfaces, where intergrain friction should be larger to start with and can be reduced even more by the small interlopers.

This conjecture is indeed confirmed by the data in Fig. 4, where we mixed the three aforementioned grains with a fourth granular material (Ottawa sand, $d_{m}=350 \mu \mathrm{m}$ ) which has a much more irregular shape with $C \approx 0.81$ [Fig. 1(b)]. All three mixtures are at $M=25 \%$ and each induces a packing fraction higher than that of the pure Ottawa sand, $\phi=56.5 \%$; however each mixture leads to a higher penetration depth. The effect is relatively small, but consistent for the mixtures involving the 178- and 520- $\mu \mathrm{m}$ grains, but quite dramatic for the $31-\mu \mathrm{m}$ grain with a doubling of the penetration depth for the higher velocities. This interesting observation is consistent with the data in Figs. 3(a), 3(b), and 3(c) and also fits with previous conclusions regarding the initial packing state relative to the critical packing state $[8,23]$.

We thus propose that lubrication in a bidisperse mixture stems from fluidization and depends on the size ratio of the two grains used in the mixture defined as $\epsilon=d_{\text {large }} / d_{\text {small }}$ [27]. In the studied Ottawa mixtures, the size ratios are $\epsilon=1.5,2$, and 11.3 for the mixtures of Ottawa sand with 520, 178, and $31 \mu \mathrm{m}$, respectively. In the glass bead mixtures, the size ratios are $\epsilon=2.9,5.7$, and 17. From inspection of the data, we could thus tentatively conclude that the size ratio must exceed a threshold of $\epsilon^{*} \approx 3$ for significant lubrication effects to take place.

\section{Effect of packing fraction}

A recent study of bidisperse mixtures [28] found that the inclusion of "rattlers" (i.e., small particles with a large size ratio between large and small particles) fills in the voids between large particles and dramatically increases the critical packing fraction. However, it is unclear how the rattlers affect the dynamical response of the granular material to impact, for example, or how they affect the interstitial forces between grains. Our study here indicates that the inclusion of fine grains into a bed of larger grains brings in the influence of ambient air and local fluidization, which has been observed previously for dense spheres impacting onto granular beds [23-25] and in falling granular streams $[29,30]$. The influence of ambient air only appears to be significant for finer grains (Geldart group A), such as the smallest particles used here with $d_{p}=31 \mu \mathrm{m}$, and is typically not present for larger grains (Geldart group B, typically with mean diameter between 40 and $500 \mu \mathrm{m}$ ), such as the larger grains used here with $d_{p}=178$ and $520 \mu \mathrm{m}$, according to Geldart's classification [31].

In order to study directly the effect of the packing fraction for a given mixture, we use $31-\mu \mathrm{m}$ grains in $178-\mu \mathrm{m}$ grains at two fixed mass fractions of $M=30 \%$ and $70 \%$, shown in Figs. 5(a) and 5(b), respectively. The increase of the packing fraction was achieved by overflowing the container with the mixture and compressing the surface with a weighted plate [13], as described in the experimental section of this paper. The results for both are consistent in that the penetration depth is reduced as the initial packing fraction increases. The most dramatic reduction is for $M=70 \%$ at $\phi=60.7 \%$, shown in Fig. 5(b), where the penetration depth is substantially reduced from the trend line for the $178-\mu \mathrm{m}$ grains.

This can also be viewed for the smaller mass fraction in Fig. 5(a), during the inclusion of small grains, where the same effect arises at a higher packing fraction. As such, this would indicate that the inclusion of larger grains into the smaller grains can dramatically reduce the penetration depth at high packing fractions.

\section{E. Combined scaling}

Given the nonlinear dependence of the packing fraction upon the mass fractions in the composite granular materials and increased or decreased penetration with higher packing fractions, a single-valued critical packing state cannot be 

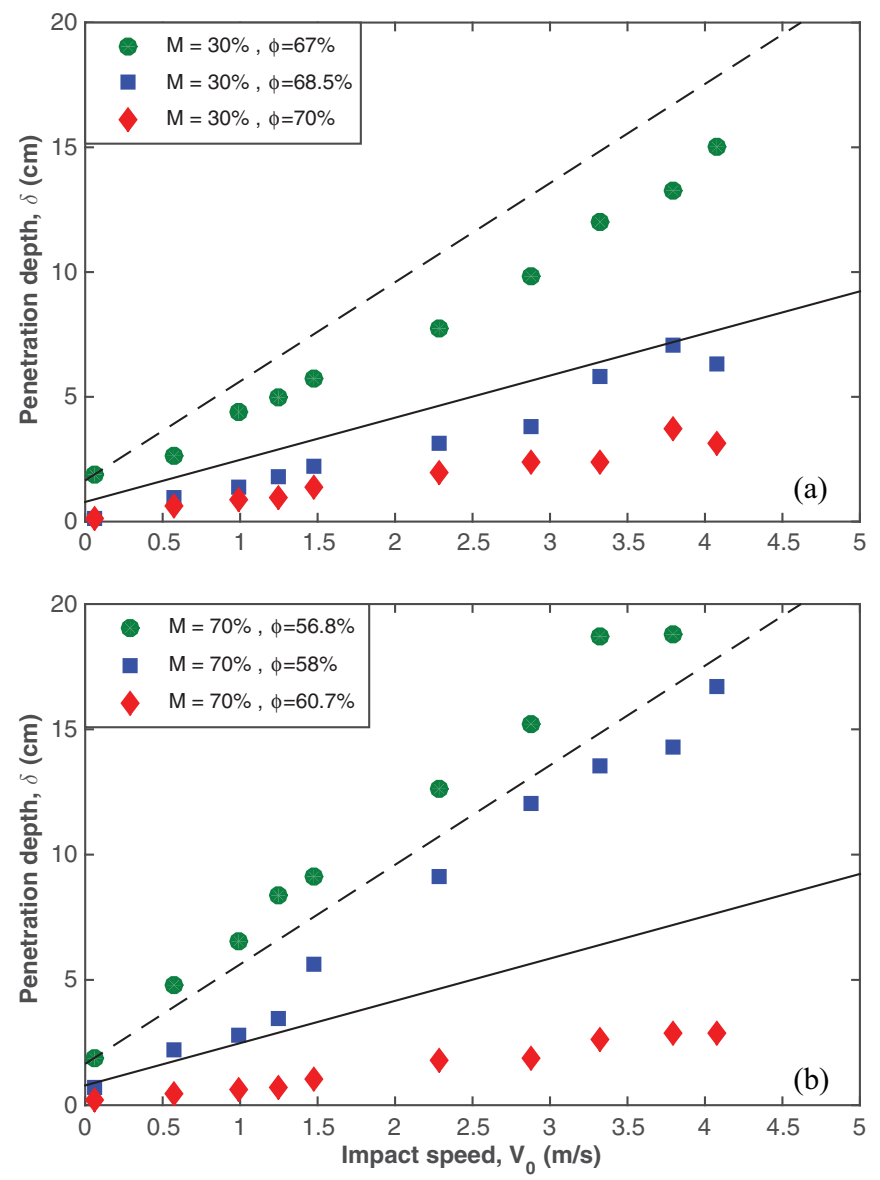

FIG. 5. Effect of packing fraction on penetration depth for $31 \mu \mathrm{m}$ glass beads mixed with $178 \mu \mathrm{m}$ of (a) $M=30 \%$ and (b) $M=70 \%$. The dashed and solid lines indicate the trend lines for pure $31 \mu \mathrm{m}$ and $178 \mu \mathrm{m}$ poured.

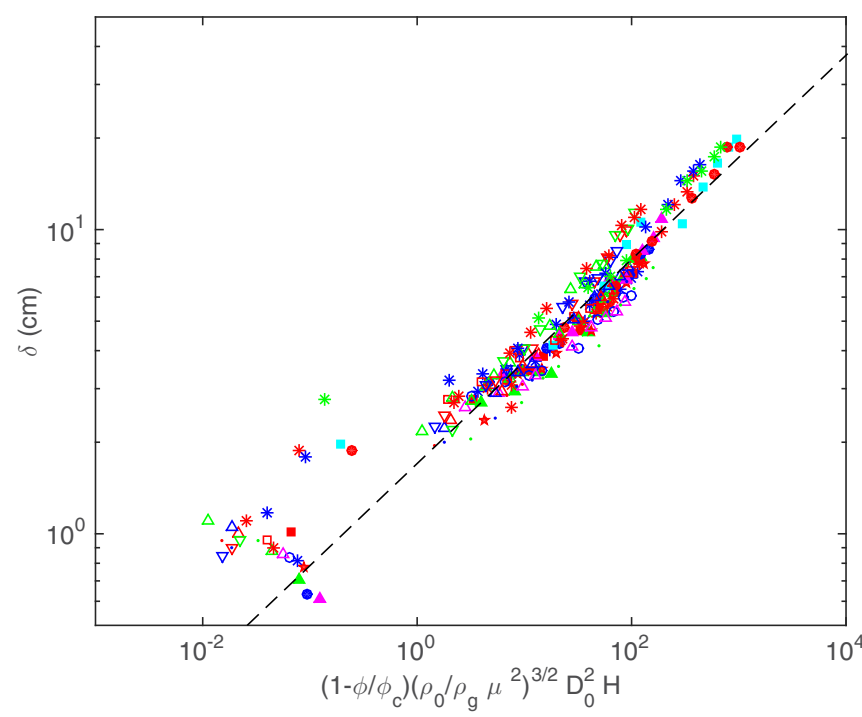

FIG. 6. Scaling of all data sphere penetration points of the bidisperse mixtures in all three combinations of the glass beads presented in Fig. 3, with the additional $\left(1-\phi / \phi_{c}\right)$ factor, where $\phi_{c}$ differs between mixtures as shown in Fig. 7. The slope of the dashed line is $1 / 3$.
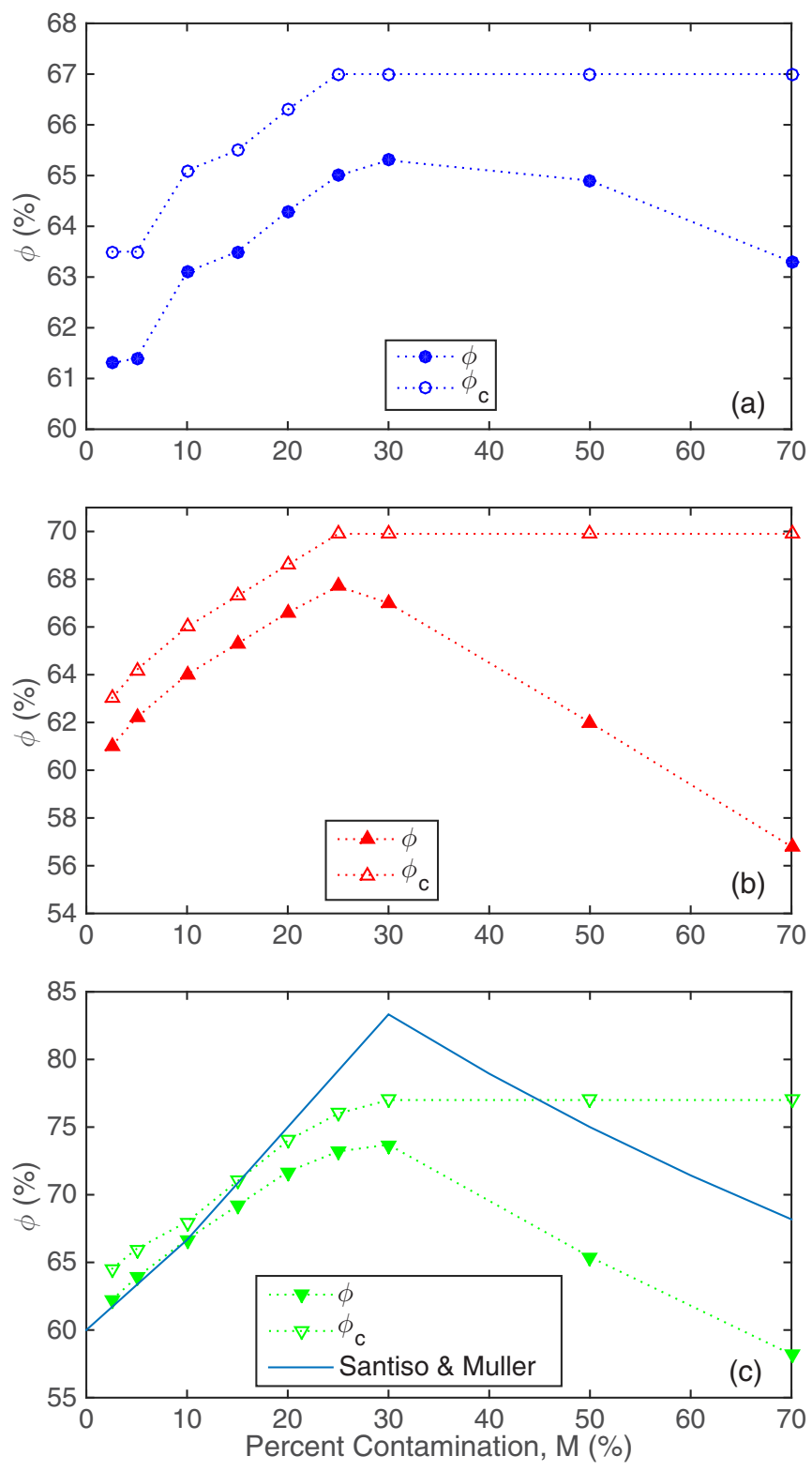

FIG. 7. Packing fractions $\phi$ and empirically deduced critical packing fraction $\phi_{c}$ for bidisperse mixtures of (a) 178 $\mu \mathrm{m}$ in $520 \mu \mathrm{m}$, (b) $31 \mu \mathrm{m}$ in $178 \mu \mathrm{m}$, and (c) $31 \mu \mathrm{m}$ in $520 \mu \mathrm{m}$.

derived for these experiments to generate scaling laws to collapse the data, such as that shown in Fig. 2(c) for monodisperse grains. Using the scaling for $H$ shown in Eq. (2) and Fig. 2(c), with a single critical packing fraction $\phi_{c}$ derived using the approach of Ref. [19] for each mixture, fails to collapse the data. Nonetheless, a single critical value for each mixture, $\phi_{c i}$, could be empirically deduced, and all the bidisperse data presented collapse while using the $1-\phi / \phi_{c i}$ factor and are shown to follow a line of slope 1/3 in Fig. 6. In this collapse, $\phi_{c}$ is now a fitting parameter, found in Fig. 7. In each mixture case, $\phi_{c i}$ is seen to initially follow the trend line of the measured packing fraction $\phi$, which was previously noted during the experiments for respective $M$ values with $\phi_{c i} \sim 2 \%$ higher than the measured values $\phi$. However, as $M$ 


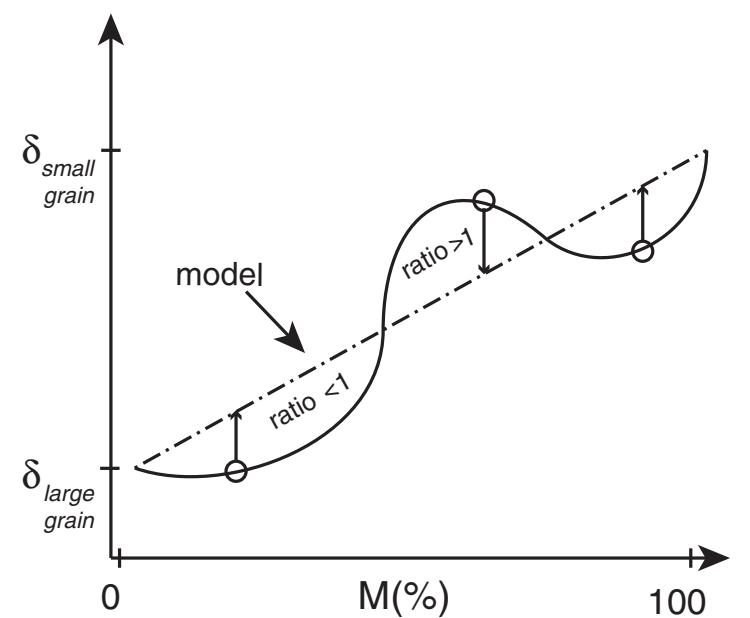

FIG. 8. Graphical representation of the interpolation model. Dash-dotted line corresponds to the interpolation model for one impact velocity value $V_{0}$ for $M$ between 0 and $100 \%$ and the solid line indicates the measurements. The ratio of measured to predicted depths, $\beta=\delta / \delta_{\text {model }}$, is plotted in the following figure. A ratio $<1$ corresponds to smaller penetration depth, and a ratio $>1$ corresponds to a fluidization effect and increased penetration.

reaches $\sim 25 \%, \phi_{c i}$ saturates to a constant value, while the experimental values of $\phi$ decrease (Fig. 7). The experimental values of $\phi$ therefore exhibit a maximum, which confirms the theoretical trend suggested by the simulation of Santiso and Müller [27]. They proposed that the value of the packing fraction in a bidisperse bed follows the equations

$$
\begin{aligned}
& \phi=\frac{\phi_{m}}{1-w_{1}\left(1-\phi_{m}\right)} \text { for } w_{1}<\frac{1}{2-\phi_{m}}, \\
& \phi=\frac{\phi_{m}}{w_{1}} \text { for } w_{1} \geqslant \frac{1}{2-\phi_{m}},
\end{aligned}
$$

where $w_{1}$ is the fraction of the larger grain size and $\phi_{m}$ is the random close packing fraction in the monodisperse case, leading to the existence of a peak at around $M=30 \%$. Note also the values of $\phi$ and $\phi_{c i}$ generally increase as the size ratio $\epsilon$ increases. The theoretical curve included in Fig. 7(c) is from Ref. [27] for the asymptotic case where the small grains are infinitely smaller than the large grains.

\section{F. Interpolation model}

Figure 3 showed that the increased lubrication effect from adding smaller grains did not grow linearly with the mass fraction of the added material. For a small amount of added material the penetration depth changed minimally, whereas over $30 \%$ it greatly increased this depth. To graphically characterize this lubrication effect, we seek to present our data using a normalized contour plot to show the influence of the mass fraction $M$ on the penetration depth. To do this, we first interpolate our data sets with a simple linear interpolation model. In this case the value of an expected penetration depth is calculated as follows. For a given impact velocity $V_{o}$, we assume that the addition of $M \%$ of the small material will linearly increase the penetration depth from the $\delta$ value for the pure large grains $(M=0 \%)$ up to the $\delta$ value for the small (a)

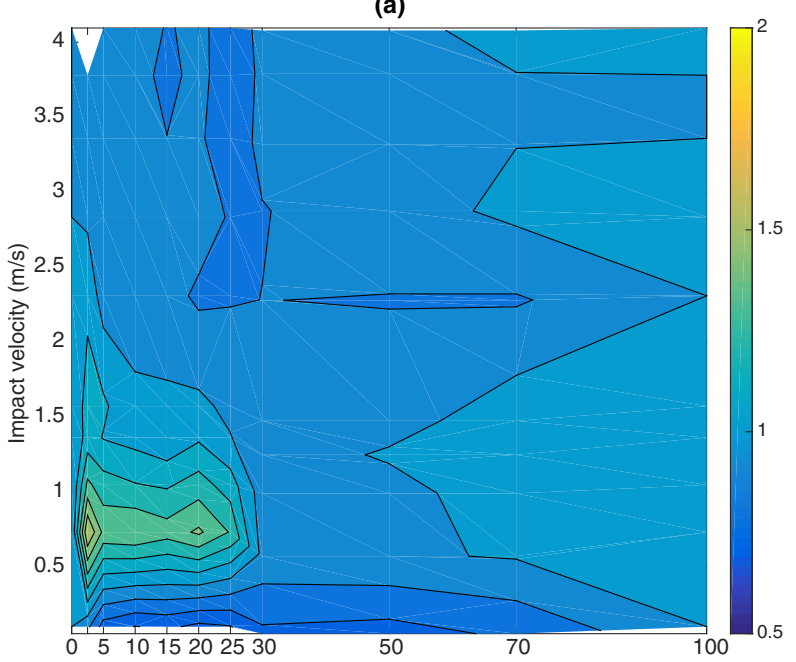

(b)

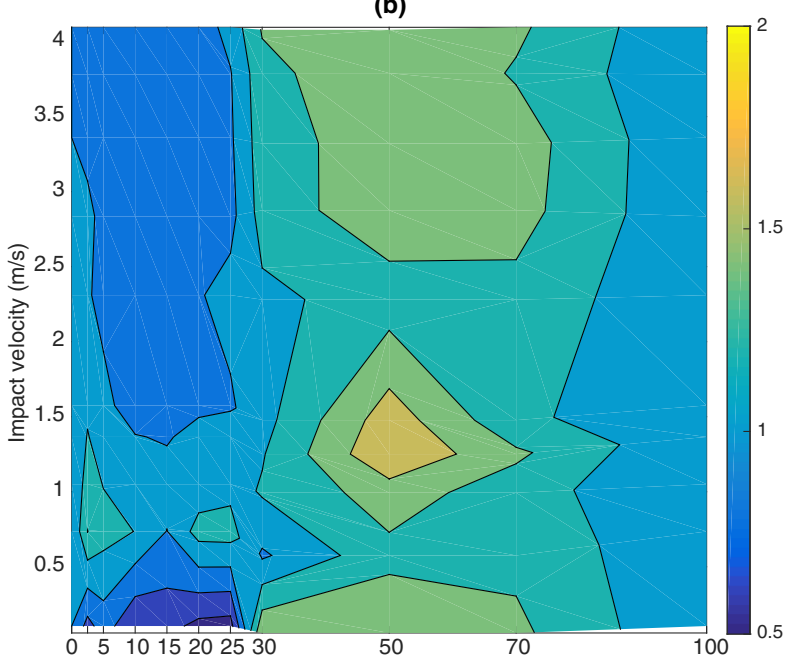

(c)

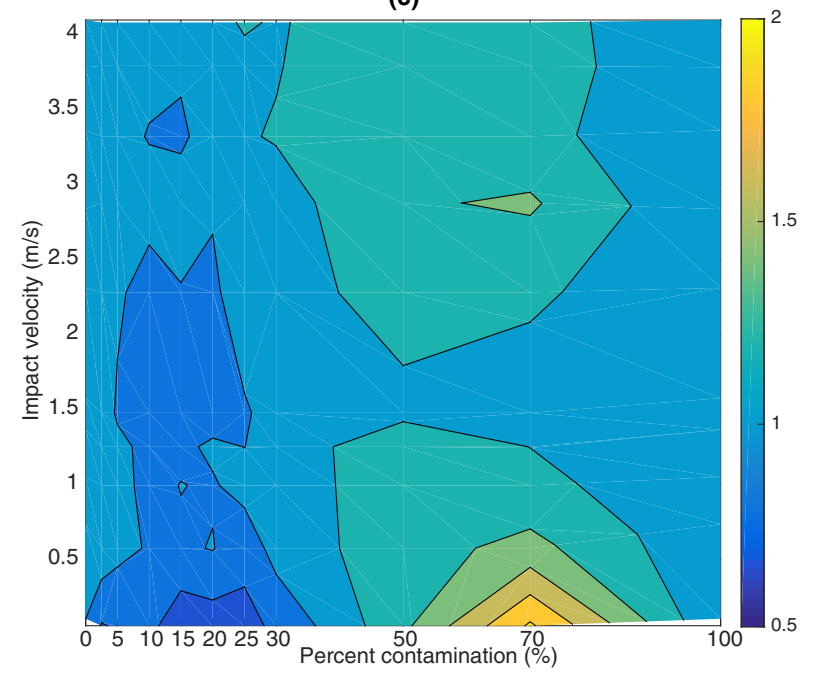

FIG. 9. Contour plot showing normalized penetration as a function of mass fraction, $M$, and impact speed, $V_{0}$, for bidisperse mixtures. The color indicates the penetration depth relative to a simple model based on a linear interpolation between the two pure grains (i.e., $M=0$ and $M=100$ ) given by Eq. (4). The contour plots represent the interpolated data for (a) $178 \mu \mathrm{m}$ in $520 \mu \mathrm{m}$, (b) $31 \mu \mathrm{m}$ in $178 \mu \mathrm{m}$, and (c) $31 \mu \mathrm{m}$ in $520 \mu \mathrm{m}$. 
grains $(M=100 \%)$. This is schematically shown in Fig. 8 and expressed mathematically as

$$
\delta_{\text {model }}=\delta_{\text {large }}+\frac{\left(\delta_{\text {small }}-\delta_{\text {large }}\right) M}{100} .
$$

We next take the ratio of the measured penetration depth $\delta$ to that predicted by this model, i.e., $\beta=\delta / \delta_{\text {model }}$. This ratio is plotted as an elevation contour in Fig. 9, for all three bidisperse combinations over the full range of impact velocities from Fig. 3. Note that a value of $\beta<1$ indicates a smaller penetration depth than the linear model, whereas $\beta>1$ indicates a larger penetration depth than expected.

The contour plots in Fig. 9 make it clear that the deviations from the linear model are larger when the ratio of the two grain sizes is larger [see Figs. 9(b) and 9(c)] than for the mixture of the grains which are closest in size to each other, i.e., for the case of 178- $\mu \mathrm{m}$ grains in 520- $\mu \mathrm{m}$ grains shown in Fig. 9(a). In Fig. 9(a) the only significant deviations are observed at a combination of low mass fractions $(M<30 \%)$ and low velocities, where $\beta \approx 1.5$ in a region of the data. On the other hand, for the cases with larger differences in grain sizes, for mixtures of $31-\mu \mathrm{m}$ grains in $178-\mu \mathrm{m}$ grains [Fig. 9(b)] and for $31-\mu \mathrm{m}$ grains in 520- $\mu \mathrm{m}$ grains [Fig. 9(c)], there is a vertical ridge of maximum lubrication effects occurring at around 50-50 mixtures for all impact velocities. In Fig. 9(b) this lubrication effect is quite pronounced with $\delta / \delta_{\text {model }}$ showing values between 60 and $85 \%$ above the linear interpolation model. In Fig. 9(c) this ridge is less pronounced.

Besides the regions of lubricating effects, Figs. 9(b) and 9(c) also show reduced penetration for mass fractions below 30\%. In this regime the work of Ref. [28] proposes that the small particles sitting in the space between the large particles are not part of the effective load-bearing chains or force networks, hence they are considered "rattlers." This comparison is particularly relevant for the large size ratios in Figs. 9(b) and 9(c) since they are close to or above the critical size ratio identified by Koeze et al. [28] of $\epsilon^{*} \approx 6.46$.

\section{CONCLUSIONS}

Herein, we have studied the penetration of impacting solid spheres into granular media, which consist of mixtures of two widely differently sized grains, i.e., bimodal media with smaller grains interspersed between larger grains.

By systematically varying the mass fraction ratios of two glass beads over a range of impact speeds $\left[V_{0}<6 \mathrm{~m} / \mathrm{s}\right.$,
$\mathrm{Fr}=V_{0}^{2} /\left(g D_{0}\right) \approx O(1-100)$, where FR is Froude number $]$, we have identified mixture regimes where the addition of the small grains "lubricates" the impact, resulting in increased sphere-penetration depths, when compared to the corresponding monodispersed medium. This lubrication occurs even though the addition of the small grains increases the overall packing fraction of the bed, but requires a sufficiently large ratio of grain sizes, which we estimate to be between $\epsilon=3$ and $\epsilon=5.7$. Furthermore, small amounts of the finer grains do not lubricate and require $M \gtrsim 30 \%$ for the effect to set in.

The penetration results could not be scaled with the packing-fraction methods used in earlier studies of monodisperse media, but rather required using different critical packing fractions for each bidisperse mixture.

Based on these results, we propose that the mechanism for lubrication is that the smaller grains get into the gaps between the larger grains, thereby propping them apart and promoting an effective lubrication by reducing the friction between the larger grains. This explains why sufficient mass fraction of smaller grains is needed, as a small number of them will simply act like rattlers, bouncing around in the voids between the larger grains, whereas above a critical mass fraction they begin to strongly influence the force chains between the large grains.

If reduced friction between the larger grains is at play, then this effect should be even more dramatic for large grains which have rough surfaces, where intergrain friction should be larger to start with and can be reduced even more by the small interlopers. This is indeed supported the data presented herein with rough Ottawa sand, where the largest lubrication effects are realized.

This work raises numerous open questions, the most important of which is how to disentangle the effects of the bidispersity and the packing fraction. The two are inherently linked and cannot be easily varied independently in experiments. Further investigations under reduced pressure would be helpful in pinpointing how the smaller grains cause the lubrication and how important the airflow is. The impact dynamics for multicomponent granular media [14,21,22,32] appears to exhibit a complex dependence on the composition of the granular bed and therefore needs to be explored further by systematic variation of the composition and the packing fraction.

\section{ACKNOWLEDGMENTS}

The research described herein was partially funded by KAUST. We acknowledge the financial support from CCRC at KAUST, Extreme Combustion FCC/1/1975.
[1] R. A. Bagnold, The Physics of Blown Sand and Desert Dunes (Dover, New York, 2004), pp. 107-124.

[2] J. Bear, Dynamics of Fluids in Porous Media (Dover, New York, 1988), pp. 38-47.

[3] J. S. Uehara, M. A. Ambroso, R. P. Ojha, and D. J. Durian, Phys. Rev. Lett. 90, 194301 (2003).

[4] A. M. Walsh, K. E. Holloway, P. Habdas, and J. R. de Bruyn, Phys. Rev. Lett. 91, 104301 (2003).
[5] J. C. Amato and R. E. Williams, Am. J. Phys. 66, 141 (1998).

[6] M. A. Ambroso, C. R. Santore, A. R. Abate, and D. J. Durian, Phys. Rev. Lett. 71, 051305 (2005).

[7] D. I. Goldman and P. Umbanhowar, Phys. Rev. E 77, 021308 (2008).

[8] P. Umbanhowar and D. I. Goldman, Phys. Rev. E 82, 010301 (2010). 
[9] L. Kondic, X. Fang, W. Losert, C. S. O'Hern, and R. P. Behringer, Phys. Rev. E 85, 011305 (2012).

[10] A. H. Clark, A. J. Petersen, and R. P. Behringer, Phys. Rev. E 89, 012201 (2014).

[11] M. Kramar, A. Goullet, L. Kondic, and K. Mischaikow, Phys. Rev. E. 90, 052203 (2014).

[12] S. T. Thoroddsen and A. Q. Shen, Phys. Fluids 13, 4 (2000).

[13] J. O. Marston, E-Q. Li, and S. T. Thoroddsen, J. Fluid Mech. 704, 5 (2012).

[14] J. O. Marston, M. M. Mansoor, and S. T. Thoroddsen, Phys. Rev. E 88, 010201(R) (2013).

[15] J. E. Colwell, S. Sture, M. Cintala et al., Icarus 195, 908 (2008).

[16] Y. Li, A. Dove, J. S. Curtis, and J. E. Colwell, Powder Technol. 288, 303 (2016).

[17] K. A. Newhall and D. J. Durian, Phys. Rev. E 68, 060301(R) (2003).

[18] H. Katsuragi and D. J. Durian, Nat. Phys. 3, 420 (2007).

[19] J. de Bruyn and A. M. Walsh, Can. J. Phys. 82, 439 (2004).

[20] A. Seguin, Y. Bertho, and P. Gondret, Phys. Rev. E 78, 010301 (2008).

[21] J. O. Marston, I. U. Vakarelski, and S. T. Thoroddsen, Phys. Rev. E 86, 020301 (2012).
[22] T. A. Brzinski, III, J. Schug, K. Mao, and D. J. Durian, Phys. Rev. E 91, 022202 (2015).

[23] J. R. Royer, B. Conyers, E. I. Corwin, P. J. Eng, and H. M. Jaeger, Europhys. Lett. 93, 28008 (2011).

[24] G. Caballero, R. Bergmann, D. van der Meer, A. Prosperetti, and D. Lohse, Phys. Rev. Lett. 99, 018001 (2007).

[25] S. Joubaud, T. Homan, Y. Gasteuil, D. Lohse, and D. van der Meer, Phys. Rev. E 90, 060201(R) (2014).

[26] J. R. Royer, E. I. Corwin, A. Flior, M. L. Cordero, M. L. Rivers, P. J. Eng, and H. M. Jaeger, Nat. Phys. 1, 164 (2005).

[27] E. Santiso and E. A. Müller, Mol. Phys. 100, 2461 (2002).

[28] D. J. Koeze, D. Vaberg, B. B. T. Tjoa, and B. P. Tighe, Euro. Phys. Lett. 113, 54001 (2016).

[29] S. R. Waitukaitis, H. F. Grutjen, J. R. Royer, and H. M. Jaeger, Phys. Rev. E. 83, 051302 (2011).

[30] J. R. Royer, D. J. Evans, L. Oyarte, Q. Guo, E. Kapit, M. E. Möbius, S. R. Waitukaitis, and H. M. Jaeger, Nature (London) 459, 1110 (2009).

[31] D. Geldart, Types of gas fluidization, Powder Technol. 7, 285 (1973).

[32] J. O. Marston, J. E. Sprittles, Y. Zhu, E.-Q. Li, I. U. Vakarelski, and S. T. Thoroddsen, Powder Technol. 239, 128 (2013). 